\title{
Transient analysis of water transport in PEM fuel cells
}

\author{
Wei-Mon Yan ${ }^{\text {a,* }}$, Hsin-Sen Chu ${ }^{\text {b }}$, Jian-Yao Chen ${ }^{b}$, \\ Chyi-Yeou Soong ${ }^{\mathrm{c}}$, Falin Chen $^{\mathrm{d}}$ \\ ${ }^{\text {a }}$ Department of Mechatronic Engineering, Huafan University, Shih-Ting, Taipei 223, Taiwan, ROC \\ ${ }^{\mathrm{b}}$ Department of Mechanical Engineering, National Chiao Tung University, Hsin-Chu 300, Taiwan, ROC \\ ${ }^{\mathrm{c}}$ Department of Aerospace and System Engineering, Feng Chia University, Seatwen, Taichung 407, Taiwan, ROC \\ ${ }^{\mathrm{d}}$ Institute of Applied Mechanics, National Taiwan University, Taipei 106, Taiwan, ROC \\ Received 5 July 2006; received in revised form 25 July 2006; accepted 25 July 2006 \\ Available online 24 August 2006
}

\begin{abstract}
This paper theoretically studies the water transport phenomena in PEM fuel cells, mainly investigating the transient behavior in the gas diffusion layer (GDL), catalyst layer (CL) and proton exchange membrane (PEM). In the PEM, both diffusion and electro-osmosis processes are considered, while in the GDL and CL, only diffusion process is taken into account. The process of water uptake is employed to account for the water transport at the interface between the PEM and CL. The results indicate that the water content in the PEM and the time for reaching the steady state in the start-up process are influenced by the humidification constant, $k$, the humidification, and the thickness of PEM. The rise of the $k$ increases the water content in the membrane and shortens the time for reaching the steady state. Insufficient humidification causes relatively small water content and long steady time. When the PEM is thinner, the water is more uniformly distributed, the water content gets higher, and the time for reaching the steady state is distinctly shorter.
\end{abstract}

(C) 2006 Elsevier B.V. All rights reserved.

Keywords: Water transport; PEM fuel cells; Transient analysis

\section{Introduction}

Water management gets to be a crucial problem in the recent research in PEM fuel cells, in which adjusting associated parameters is an important consideration. Okada demonstrated a series of studies on water transport and distribution in the electrolyte membrane of fuel cells to investigate the influence of the membrane characteristics, operating conditions, and contamination. An one-dimensional mathematical model involving water diffusion and electro-osmotic process in a membrane to discuss the water content profile under various operating conditions was proposed by Okada et al. [1]. The results indicated that the water content profile was greatly influenced by the electrical current density and water transfer coefficient. In 1998, Okada et al. [2,3] experimentally and analytically investigated the effects of contamination on water transport in a half membrane of the anode side. They found that, when the PEM was polluted, the water

\footnotetext{
* Corresponding author. Tel.: +886 26632102; fax: +886226632143. E-mail address: wmyan@huafan.hfu.edu.tw (W.-M. Yan).
}

molecules were dragged by the contaminator rather than hydrogen ions, which caused the drop of electrical current density. Okada et al. [4] extended the previous model to the whole membrane, and conducted some calculations according to the actual experimental conditions. Later, Okada et al. $[5,6]$ theoretically analyzed the influence of contamination on the whole membrane characteristics in the conditions of contaminated anode input, and the cathode input with finite thickness of contaminated region.

Eikerling et al. [7] presented a model to predict the water transport process under the electro-osmosis drag and capillary force. Bernardi and Verbrugge [8] put forward a model to describe the physical process in the PEM, CL and GDL on the cathode side, mainly investigating the cathode polarization, the water transport in the PEM and CL, and the utilization ratio of the CL. Bernardi and Verbrugge [9] examined the mass transport and reaction process in the region including the anode CL, PEM, cathode CL and cathode GDL. Singh et al. [10] further extended the one-dimensional model of Ref. [9] into two-dimensional model to analyze the dimensional effects. It was pointed out that the voltage resulted from the two-dimensional model was appre- 


\begin{tabular}{|c|c|}
\hline \multicolumn{2}{|c|}{ Nomenclature } \\
\hline$a$ & water activity \\
\hline$c_{i, j}$ & water concentration in each layer $\left(\mathrm{mol} \mathrm{cm}^{-3}\right)$ \\
\hline$D^{m}$ & water diffusivity in PEM $\left(\mathrm{cm}^{2} \mathrm{~s}^{-1}\right)$ \\
\hline$D^{(0)}$ & $\begin{array}{l}\text { the constant term in Taylor series of water diffu- } \\
\text { sivity in PEM }\left(\mathrm{cm}^{2} \mathrm{~s}^{-1}\right)\end{array}$ \\
\hline$D^{(1)}$ & $\begin{array}{l}\text { the first order term in Taylor series of water dif- } \\
\text { fusivity in PEM }\left(\mathrm{cm}^{2} \mathrm{~s}^{-1}\right)\end{array}$ \\
\hline$D_{\mathrm{w}}^{\mathrm{b}}$ & $\begin{array}{l}\text { water vapor diffusivity in gas diffusion layer } \\
\left(\mathrm{cm}^{2} \mathrm{~s}^{-1}\right)\end{array}$ \\
\hline$D_{\mathrm{w}}^{\mathrm{k}}$ & water vapor diffusivity in catalyst layer $\left(\mathrm{cm}^{2} \mathrm{~s}^{-1}\right)$ \\
\hline$F$ & Faraday's constant $\left(\mathrm{C} \mathrm{mol}^{-1}\right)$ \\
\hline I & electrical current density $\left(\mathrm{Acm}^{-2}\right)$ \\
\hline$J_{\mathrm{H}_{2} \mathrm{O}}$ & water flux $\left(\mathrm{mol} \mathrm{cm}^{-2} \mathrm{~s}^{-1}\right)$ \\
\hline$k$ & humidification constant $\left(\mathrm{cm} \mathrm{s}^{-1}\right)$ \\
\hline$M_{\mathrm{w}}$ & molecular weight of water $\left(\mathrm{g} \mathrm{mol}^{-1}\right)$ \\
\hline$P$ & pressure \\
\hline $\bar{r}$ & mean pore size $(\mathrm{cm})$ \\
\hline$R$ & universal gas constant $\left(\mathrm{J} \mathrm{mol} \mathrm{K}^{-1}\right)$ \\
\hline$w$ & water transfer coefficient \\
\hline$w^{(0)}$ & $\begin{array}{l}\text { the constant term in Taylor series of water transfer } \\
\text { coefficient in PEM }\end{array}$ \\
\hline$w^{(1)}$ & $\begin{array}{l}\text { the first-order term in Taylor series of water trans- } \\
\text { fer coefficient in PEM }\end{array}$ \\
\hline \multicolumn{2}{|c|}{ Greek letters } \\
\hline$\varepsilon_{\mathrm{c}}$ & porosity of catalyst layer \\
\hline$\varepsilon_{\mathrm{d}}$ & porosity of gas diffusion layer \\
\hline$\xi$ & electrochemical reaction rate $\left(\mathrm{A} \mathrm{cm}^{-3}\right)$ \\
\hline$\zeta_{j}$ & molar fraction of specie $j$ \\
\hline$\lambda$ & water content in membrane $\left(\mathrm{mol} \mathrm{H}_{2} \mathrm{O} / \mathrm{mol} \mathrm{SO}_{3}^{-}\right)$ \\
\hline \multicolumn{2}{|c|}{ Subscripts } \\
\hline $\mathrm{c}$ & catalyst layer \\
\hline $\mathrm{d}$ & gas diffusion layer \\
\hline $\mathrm{g}$ & gas \\
\hline 1 & liquid \\
\hline $\mathrm{m}$ & proton exchange membrane \\
\hline
\end{tabular}

ciably less than that from the one-dimensional model. Springer et al. [11] proposed a one-dimensional isothermal model to analyze the water transport, and found that the ratio of water molecule flux to hydrogen ion flux was quite less than the directly measured electro-osmosis coefficient. Springer et al. [12] further indicated that, when the cathode fuel was air, the potential loss of PEM was only a small part of the overall loss, which mainly occurred in the cathode. Fuller and Newman [13] theoretically investigated the water and thermal management in fuel cells. Bradean et al. [14] presented a model of a half fuel cell on the cathode side to discuss the influence of operating conditions on the cell performance. They indicated that the diffusion process was the main mode of the mass transport, and the occurrence of super saturation of water vapor was definitely associated with the cathode thickness and the reaction rate.
Nguyen and White [15] developed a one-dimensional model to investigate the water transport in the membrane under the diffusion and electro-osmosis processes, coupled with the heat transfer between the solid and gas phases, involving the latent heat absorbed/emitted in evaporation/condensation. The results showed that, when the current density was over $1 \mathrm{~A} \mathrm{~cm}^{-2}$, the Ohmic loss in the membrane played an important role in the overall potential loss. Yi and Nguyen [16] improved the model in Ref. [15] to involve the convection process induced by the pressure gradient, and analyzed the temperature profile along the flow passages. Later, He et al. [17] proposed a two-dimensional model to study the two-phase flow phenomena in fuel cells. To clarify the water effects, Yan et al. [18] examined the effects of flow distributor geometry and diffusion layer porosity on reactant gas transport and performance of PEM fuel cells. In Ref. [18], the porosity of gas diffusion layer was varied to account the effects of water content. They found that the porosity of gas diffusion layer has significant impact on the cell performance.

The water content in the membrane affects the electrical resistance to influence the cell performance. An experimental study on Nafion 117 membrane performance on water transport at $30^{\circ} \mathrm{C}$ was conducted by Zawodzinski et al. [19]. Hinatsu et al. [20] also performed an experiment to investigate the water uptake process, and obtained the performance of membrane submerged in the liquid water at a temperature from 25 to $130^{\circ} \mathrm{C}$. Futerko and Hsing [21] deduced a model to predict the water content variation in the membrane, and compared the results with the available experimental results. Recently, the dynamic performance of PEM fuel cell was examined by Chen et al. [22]. In their work, the transient behaviors of the water transport across the membrane were examined, as well as the influences of several physical parameters on the characteristic time to reach the steady state. The detailed thermal and water management in the membrane of PEM fuel cells is investigated numerically by Yan et al. [23]. The coupling effects of mass diffusion and temperature gradient on the water distribution of the membrane were taken into account with consideration of the temperaturedependent diffusivity.

The literature, as described above, was mainly restricted in the steady-state performance and mass distribution in the fuel cell, but lacks the transient analysis. The present work aims to investigate the transient process to obtain the time for reaching the steady situation and to understand the evolution of water content under various conditions. A one-dimensional transient model is further developed by adding the mass transport process in GDL and CL into the steady model derived by Okada's research group, which only considered the transport process in the membrane.

\section{Theoretical analysis}

\subsection{Assumptions}

In the present work, a systematically parametric study is conducted to examine the transient behavior of the water content across the GDL, CL and membrane under the influences of various physical parameters. A special attention is paid to the time 
needed for the water distribution across the system to reach the steady state, denoted as $t_{\mathrm{s}}$, under influence of different physical parameters. Since the thicknesses of the PEM, GDL and CL are quite small comparing to the length and width, the fuel cell is regarded as a one-dimensional system. For convenience, other assumptions are made as the following:

(1) The system is one-dimensional and isothermal.

(2) In the CL and GDL, the diffusion is the only mass transport mode.

(3) The membrane, CL and GDL are homogeneous.

(4) The electrochemical reaction only occurs in the CL on the cathode side.

(5) In the GDL and CL, water only exists in vapor phase, and could be supersaturated.

\subsection{Governing equations}

The physical model is schematically shown in Fig. 1. The origin is set at the border of the anode GDL, which is away from the interface between the anode GDL and CL at a distance of $A$, away from the interface between the anode CL and PEM at $B$, away from the interface between the cathode CL and PME at $C$, away from the interface between the cathode CL and GDL at $D$, and away from the border of cathode GDL at $E$. The mass transport equations in the GDL, CL and PEM are expressed below. Although the theoretical model is essentially an extension from Okada [1-7], we still present the details of the mathematical for-

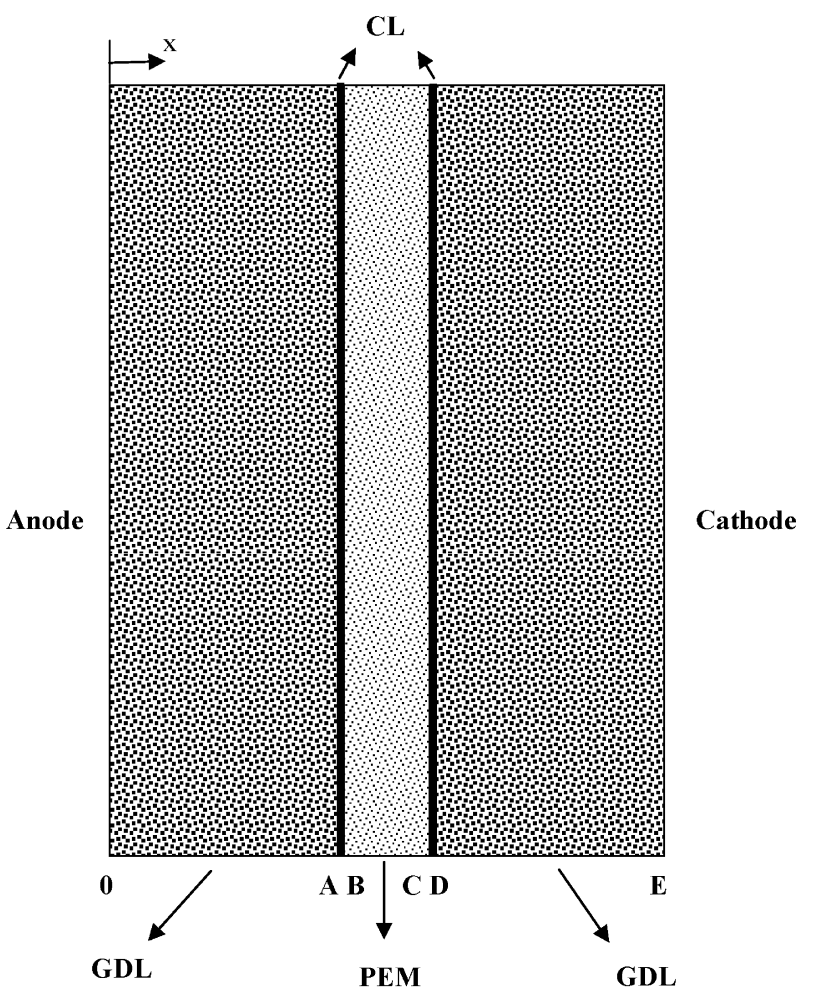

Fig. 1. Schematic diagram of the physical system. mulations and relevant physical parameters in this section for the convenience of following discussions.

As the water transport in the PEM is induced by the diffusion and electro-osmosis processes, the water flux in the PEM is described as the following:

$J_{\mathrm{H}_{2} \mathrm{O}}=-D^{m} \frac{\partial c_{3,1}}{\partial x}+\frac{I}{F} w$

where $D^{m}, c_{3,1}, I$, and $w$ denote, respectively, the water diffusivity coefficient, water content, current density, and water transfer coefficient of the PEM due to the electro-osmosis drag. And $F$ is Faraday's constant. The water content evolution in the membrane is expressed as below:

$\frac{\partial c_{3,1}}{\partial t}=-\frac{\partial J_{\mathrm{H}_{2} \mathrm{O}}}{\partial x}=\frac{\partial}{\partial x}\left(D^{m} \frac{\partial c_{3,1}}{\partial x}-\frac{I}{F} w\right)$

Since the water diffusivity and water transfer coefficient vary with water content in the membrane, they are expressed in the forms of Taylor series:

$D^{m}=D^{(0)}+D^{(1)} c_{3,1}+D^{(2)} c_{3,1}^{2}+\cdots$

$w=w^{(0)}+w^{(1)} c_{3,1}+w^{(2)} c_{3,1}^{2}+\cdots$

Substituting Eqs. (3) and (4) into Eq. (2) yields:

$$
\begin{aligned}
\frac{\partial c_{3,1}}{\partial t}= & {\left[D^{(0)} \frac{\partial^{2} c_{3,1}}{\partial^{2} x}-\frac{I}{F} w^{(1)} \frac{\partial c_{3,1}}{\partial x}\right] } \\
+ & {\left[D^{(1)} c_{3,1} \frac{\partial^{2} c_{3,1}}{\partial^{2} x}+D^{(1)}\left(\frac{\partial c_{3,1}}{\partial x}\right)^{2}\right.} \\
& \left.-\frac{2 I}{F} w^{(2)} c_{3,1} \frac{\partial c_{3,1}}{\partial x}\right]+\cdots
\end{aligned}
$$

In Eq. (5), the order of terms in the first pair of square bracket is much higher than that in the second one, thus the terms in the second square brackets are neglected. Then the equation is reduced into the form:

$\frac{\partial c_{3,1}}{\partial t}=D^{(0)} \frac{\partial^{2} c_{3,1}}{\partial^{2} x}-\frac{I}{F} w^{(1)} \frac{\partial c_{3,1}}{\partial x}$

According to Eq. (6), the water diffusivity can be assumed constant, and the water transfer coefficient should include the constant and first order terms of the Taylor's series.

In the GDL, only diffusion process is taken into account to describe the water vapor transport. As the mean pore size in the GDL is far larger than the mean free path of the water vapor molecules, it is appropriate to express the transport process as a binary diffusion process. Then the water vapor flux in the GDL is described as below:

$J_{\mathrm{H}_{2} \mathrm{O}}=-D_{\mathrm{w}}^{\mathrm{b}} \nabla c_{i, \mathrm{~g}}$

where $D_{\mathrm{w}}^{\mathrm{b}}$ is calculated from the following equation:

$\frac{\varepsilon_{\mathrm{d}}^{1.5}}{D_{\mathrm{w}}^{\mathrm{b}}}=\sum_{j} \frac{\zeta_{j}}{D_{\mathrm{w} j}^{\mathrm{b}}}$ 
where $\varepsilon_{\mathrm{d}}$ is the porosity of the GDL, $\zeta_{j}$ the molar fraction of specie $j$, and $D_{\mathrm{w} j}^{\mathrm{b}}$ is the binary diffusion coefficient of water vapor and specie $j$.

Substituting Eq. (8) into the mass conservation equation yields the diffusion equations of water vapor in the GDL:

$\varepsilon_{\mathrm{d}} \frac{\partial c_{1, \mathrm{~g}}}{\partial t}=\frac{\partial}{\partial x}\left[D_{\mathrm{w}}^{\mathrm{b}} \frac{\partial c_{1, \mathrm{~g}}}{\partial x}\right], \quad 0<x<A$

$\varepsilon_{\mathrm{d}} \frac{\partial c_{5, \mathrm{~g}}}{\partial t}=\frac{\partial}{\partial x}\left[D_{\mathrm{w}}^{\mathrm{b}} \frac{\partial c_{5, \mathrm{~g}}}{\partial x}\right], \quad D<x<E$

where $c_{1, \mathrm{~g}}$ and $c_{5, \mathrm{~g}}$ are the water vapor content in the anode GDL and cathode GDL, respectively.

Similarly, only diffusion process is considered in describing the water vapor transport in the CL. As the pore size in CL is relatively small, the collision mainly occurs between the water molecules and the wall, which is quite different from the situation in GDL. Therefore, Knudsen diffusion is employed to describe the diffusion, and then the water vapor flux in the CL is obtained:

$J_{\mathrm{H}_{2} \mathrm{O}}=-D_{\mathrm{w}}^{\mathrm{k}} \nabla c_{i, \mathrm{~g}}$

where Knudsen diffusion coefficient can be calculated by the following expression:

$D_{\mathrm{w}}^{\mathrm{k}}=\psi \bar{r} \sqrt{\frac{8 R T}{\pi M_{\mathrm{w}}}}$

where $\psi$ is the correction factor, $\bar{r}$ the mean pore size, $M_{\mathrm{W}}$ the water vapor molecular weight, and $R$ is the universal gas constant.

Letting the mass conservation replaced by Eq. (11), it becomes:

$\varepsilon_{\mathrm{c}} \frac{\partial c_{2, \mathrm{~g}}}{\partial t}=\frac{\partial}{\partial x}\left[D_{\mathrm{w}}^{\mathrm{k}} \frac{\partial c_{2, \mathrm{~g}}}{\partial x}\right], \quad A<x<B$

It is presumed that the water vapor generated from the electrochemical reaction is uniformly distributed in the cathode CL. Then the diffusion equation becomes:

$\varepsilon_{\mathrm{c}} \frac{\partial c_{4, \mathrm{~g}}}{\partial t}=\frac{\partial}{\partial x}\left[D_{\mathrm{w}}^{\mathrm{k}} \frac{\partial c_{4, \mathrm{~g}}}{\partial x}\right]+\frac{\xi}{2 F}, \quad C<x<D$

where $\varepsilon_{\mathrm{c}}$ is the porosity of the $\mathrm{CL}, c_{2, \mathrm{~g}}$ and $c_{4, \mathrm{~g}}$, respectively, denote the water vapor concentrations in the anode CL and cathode $\mathrm{CL}$, and $\xi$ is the electrochemical reaction rate.

\subsection{Initial conditions}

The initial water concentration profiles in the PEM, CL and GDL are assumed to be the steady-state distributions in the previous operation, $c_{i, j}^{0}(x)$ :

$t=0: \quad c_{i, j}(x, 0)=c_{i, j}^{0}(x)$

when $i=1,2,4,5, j=\mathrm{g}$, corresponding to the state in the GDL and CL. When $i=3, j=1$, corresponding to the state in the membrane.

\subsection{Boundary conditions}

The relative humidity is specified as constant in the two outer borders of the physical model. In the interfaces between the GDL and CL, the water concentration and water flux are continuous. In the interface between the CL and the PEM, the water transport is treated with the water uptake process. The water content in the PEM is relevant to the water activity of the surrounding water vapor, which is defined as below:

$a=\frac{c_{i, \mathrm{~g}}}{c_{\mathrm{g}}^{\mathrm{sat}}}$

where $c_{\mathrm{g}}^{\text {sat }}$ represents the saturated water vapor concentration under a certain temperature. The water content in the membrane is obtained by sorption isotherm $\Lambda(a)$ :

$\lambda \equiv \frac{c_{3,1}}{c_{\mathrm{H}^{+}}}=\Lambda(a)$

where $c_{\mathrm{H}^{+}}$is the proton molar concentration in the membrane, and $\Lambda(a)$ is calculated by the following empirical formula, which is derived from the experimental results for Nafion 117 at $80^{\circ} \mathrm{C}$ :

$$
\begin{aligned}
\Lambda= & 0.3+6 a[1-\tanh (a-0.5)]+3.9 \sqrt{a} \\
& {\left[1+\tanh \left(\frac{a-0.89}{0.23}\right)\right] }
\end{aligned}
$$

Generally, the boundary conditions of the model are listed as below:

$x=0: \quad c_{1, \mathrm{~g}}=c_{\text {in }}$

$x=A: \quad c_{1, \mathrm{~g}}=c_{2, \mathrm{~g}}$

$x=A: \quad D_{\mathrm{w}}^{\mathrm{b}} \frac{\partial c_{1, \mathrm{~g}}}{\partial x}=D_{\mathrm{w}}^{\mathrm{k}} \frac{\partial c_{2, \mathrm{~g}}}{\partial x}$

$x=B: \quad D_{\mathrm{w}}^{\mathrm{k}} \frac{\partial c_{2, \mathrm{~g}}}{\partial x}=k\left[C a-c_{3,1}\right]$

$x=B: \quad k\left[C a-c_{3,1}\right]=-D^{(0)} \frac{\partial c_{3,1}}{\partial x}+\frac{i}{F}\left[w^{(0)}+w^{(1)} c_{3,1}\right]$

$x=C: \quad k\left[C c-c_{3,1}\right]=D^{(0)} \frac{\partial c_{3,1}}{\partial x}-\frac{i}{F}\left[w^{(0)}+w^{(1)} c_{3,1}\right]$

$x=C: \quad D_{\mathrm{w}}^{\mathrm{k}} \frac{\partial c_{4, \mathrm{~g}}}{\partial x}=k\left[C c-c_{3,1}\right]$

$x=D: \quad c_{4, \mathrm{~g}}=c_{5, \mathrm{~g}}$

$x=D: \quad D_{\mathrm{w}}^{\mathrm{k}} \frac{\partial c_{4, \mathrm{~g}}}{\partial x}=D_{\mathrm{w}}^{\mathrm{b}} \frac{\partial c_{5, \mathrm{~g}}}{\partial x}$

$x=E: \quad c_{5, \mathrm{~g}}=c_{\text {in }}$

where $c_{\text {in }}$ denotes the specified water concentration, $k$ the rate constant of Henry's law accounting for the water flux entering or exiting the membrane driven by the water concentration gradient in the PEM, which is also called the humidification parameter. 
Table 1

Values of parameters used in this work

\begin{tabular}{|c|c|c|}
\hline Parameter & Symbol & Value \\
\hline Pressure at anode side (atm) & $P_{\mathrm{a}}$ & 3 \\
\hline Pressure at cathode side (atm) & $P_{\mathrm{c}}$ & 3 \\
\hline Cell temperature $\left({ }^{\circ} \mathrm{C}\right)$ & $T_{\text {cell }}$ & 80 \\
\hline Thickness of GDL (cm) & $L_{\mathrm{d}}$ & 0.03 \\
\hline Thickness of CL (cm) & $L_{\mathrm{c}}$ & 0.002 \\
\hline Thickness of PEM (cm) & $L_{\mathrm{m}}$ & 0.01 \\
\hline Effective porosity of GDL & $\varepsilon^{\mathrm{d}}$ & 0.4 \\
\hline Effective porosity of CL & $\varepsilon^{\mathrm{c}}$ & 0.3 \\
\hline Effective porosity of PEM & $\varepsilon^{\mathrm{m}}$ & 0.28 \\
\hline The constant term in Taylor series of water diffusivity in PEM $\left(\mathrm{cm}^{2} \mathrm{~s}^{-1}\right)$ & $D^{(0)}$ & $1.25 \times 10^{-5}$ \\
\hline The constant term in Taylor series of water transfer coefficient in PEM & $w^{(0)}$ & 0.0 \\
\hline The first-order term in Taylor series of water transfer coefficient in PEM & $w^{(1)}$ & 128.32 \\
\hline Water vapor diffusivity in gas diffusion layer $\left(\mathrm{cm}^{2} \mathrm{~s}^{-1}\right)$ & $D_{\mathrm{w}}^{\mathrm{b}}$ & $2.13 \times 10^{-5}$ \\
\hline Water vapor diffusivity in catalyst layer $\left(\mathrm{cm}^{2} \mathrm{~s}^{-1}\right)$ & $D_{\mathrm{w}}^{\mathrm{k}}$ & $1.39 \times 10^{-5}$ \\
\hline
\end{tabular}

$C a$ and $C c$, respectively, represent the liquid water concentrations at the interfaces between the PEM and anode CL, and between the PEM and the cathode CL, which are at equilibrium with the liquid water concentrations in the CL. $C_{\mathrm{a}}$ and $C_{\mathrm{c}}$ are calculated from the following expressions:

$$
\begin{aligned}
& C a=c_{\mathrm{H}^{+}} \Lambda\left(\frac{\left.c_{2, \mathrm{~g}}\right|_{x=B}}{c_{\mathrm{w}}^{\mathrm{sat}}}\right) \\
& C c=c_{\mathrm{H}^{+}} \Lambda\left(\frac{\left.c_{4, \mathrm{~g}}\right|_{x=C}}{c_{\mathrm{w}}^{\mathrm{sat}}}\right)
\end{aligned}
$$

\section{Results and discussions}

Based on the theoretical model with boundary and initial conditions shown in the above section, we perform a systematically parametric study to examine the transient behavior of the water content across the GDL, CL and membrane under influences of various physical parameters. These parameters include the current density, $i$, the initial current density, $i_{0}$, the relative humidity of water vapor on the cathode or anode side, the thickness of PEM, and the humidification parameter, $k$. The values of these parameters employed in the theoretical model are corresponding to the base case at $T_{\text {cell }}=80^{\circ} \mathrm{C}$, $P=3 \mathrm{~atm}, L_{\mathrm{m}}=0.01 \mathrm{~cm}, i_{0}=0 \mathrm{~A} \mathrm{~cm}^{-2}, k=0.01 \mathrm{~cm} \mathrm{~s}^{-1}$, and relative humidity $100 \%$. When the physical effect of certain parameter is evaluated, the parameter varies with the others fixed the same with the base case. The parameters used in this work are shown in Table 1.

Fig. 2 presents the steady-state distributions of the water content under four different operating current densities, with the humidification parameter $k 0.01 \mathrm{~cm} \mathrm{~s}^{-1}$, the relative humidity of water vapor on the cathode and anode sides $100 \%$, and the thickness of PEM $0.01 \mathrm{~cm}$. When a content difference exists between the PEM and its outside, $k$ denotes the magnitude of the equilibrium rate. The left figure in Fig. 2 shows the distributions of water vapor content in the anode GDL and CL, the middle one shows the distribution of liquid water content in the PEM, and
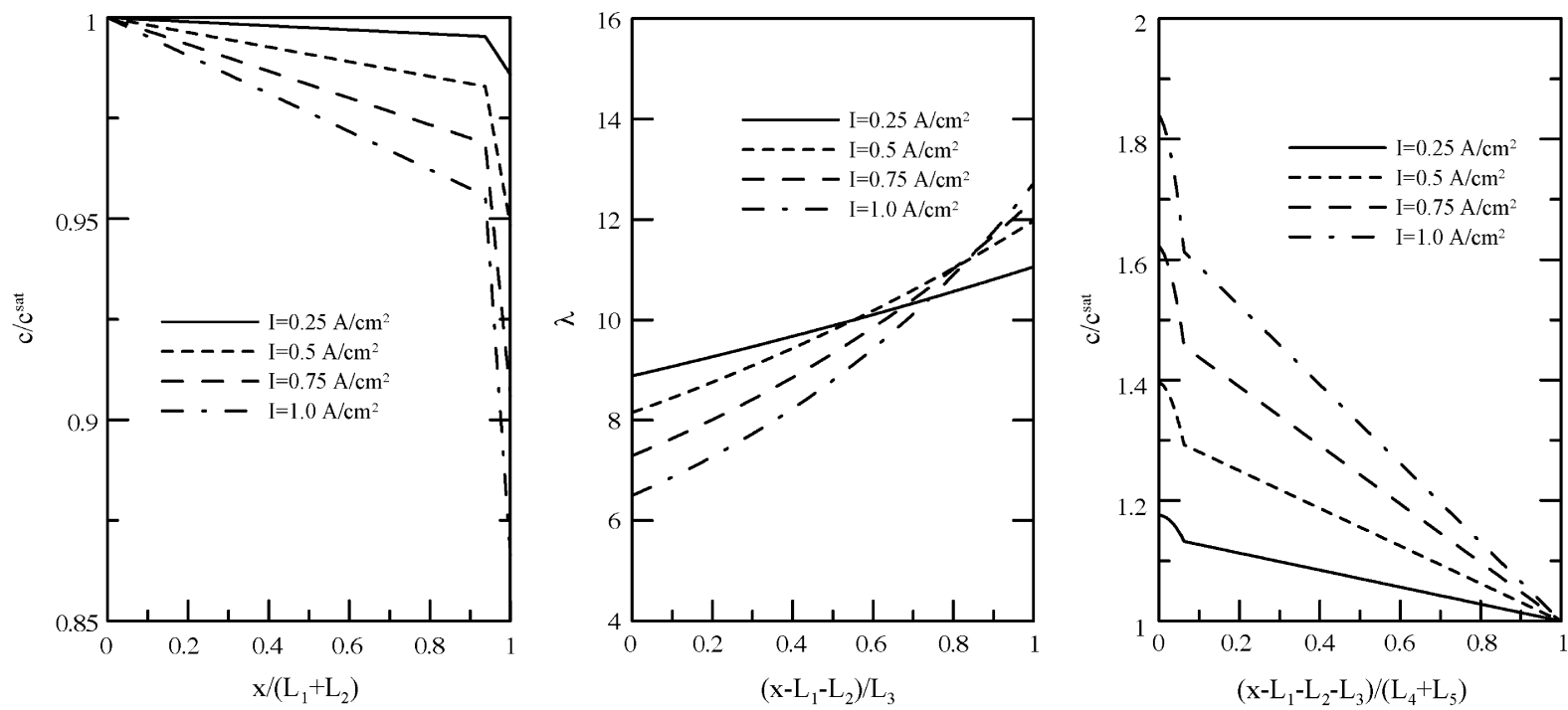

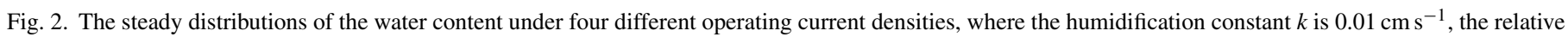
humidity of water vapor on the cathode and anode sides $100 \%$, and the thickness of PEM $100 \mu \mathrm{m}$. 
the right one indicates the distributions of water vapor content in the cathode GDL and CL. Inside the PEM, the water content near the anode side is relatively low due to the electro-osmotic effects. As the current density $i$ increasing, the electro-osmotic effects are enhanced, and the water content difference between the two sides of the PEM becomes more evident. Since the water content near the anode side of PEM is relatively low, the water vapor content in anode GDL and CL will be reduced to compensate the water depletion in PEM. The phenomena in the cathode are just opposite. The water content near the cathode side of PEM is relatively high, and the electrochemical reaction in cathode CL generates liquid water. Therefore, the water content in cathode CL is higher than that of the cathode GDL. In a separate numerical simulation, it is found that when the humidification parameter $k$ is small, the flux of the water vapor absorbed by the PEM is low, resulting high the water content in anode CL.

Fig. 3(a) and (b) presents the evolution of the water content in PEM from steady distribution at $i_{0}=0.1 \mathrm{~A} \mathrm{~cm}^{-2}$ with humidification constant being 0.01 and $0.001 \mathrm{~cm} \mathrm{~s}^{-1}$, respectively, when the operating current jumped to $i=0.1 \mathrm{~A} \mathrm{~cm}^{-2}$. It is clear from Fig. 3(a) that after the sudden change in the current density, the water content in PEM varies significantly during the initial period, since the electro-osmotic effects decrease the water content in the anode and increase that in the cathode. After about $1 \mathrm{~s}$, the water content near the cathode side approaches to the steady-state value, while the water content near the anode side continuously declines with time until it approaches the steady state. Comparison of corresponding curves between Fig. 3(a) and (b) reveals that, as humidification parameter $k$ is increased, the water content near the cathode rises and quickly approaches the steady state. This conclusion can be plausibly drawn by noting the fact that a larger $k$ implies a higher water transport performance between the PEM and its outside, which can improve the response rate of water transport.

The effects of humidification on both cathode and anode sides on the evolution of water content in the PEM are shown in Fig. 4(a) and (b), which correspond to the relative humidity of $80 \%$ and $60 \%$, respectively. According to Fig. 4(a), in the initial period, the water content near the anode side decreases due to the electro-osmotic effects, while the water content increases near the cathode side. After $2 \mathrm{~s}$, however, the back diffusion effects begin to emerge for the enlarged water content difference between the cathode and anode sides. And the water content near the anode side gradually increases. These phenomena are more obviously shown in Fig. 4(b), for the relative humidity of water vapor on the cathode and anode sides equal to $60 \%$. It is found that, when the relative humidity on the anode side is insufficient, the water content distribution in PEM varies slowly because the back diffusion process dominates, thus a relatively longer time is needed for the system with a lower relative humidity to reach the steady state.

The evolutions of the water content in the PEM at relative humidity of water vapor of $80 \%$ and $60 \%$ on the cathode side are, respectively, presented in Fig. 5(a) and (b), with the relative humidity of water vapor on the anode fixed to be $80 \%$. It is clearly seen from Fig. 5 that when the water content near the cathode side decreases, the transient response slows down near

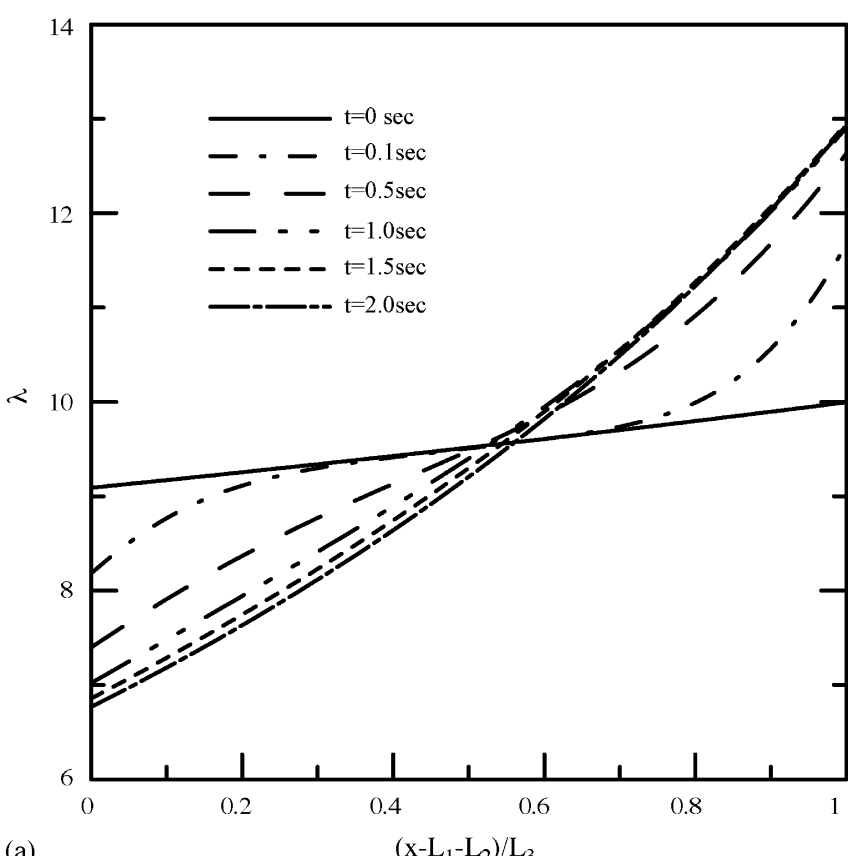

(a)

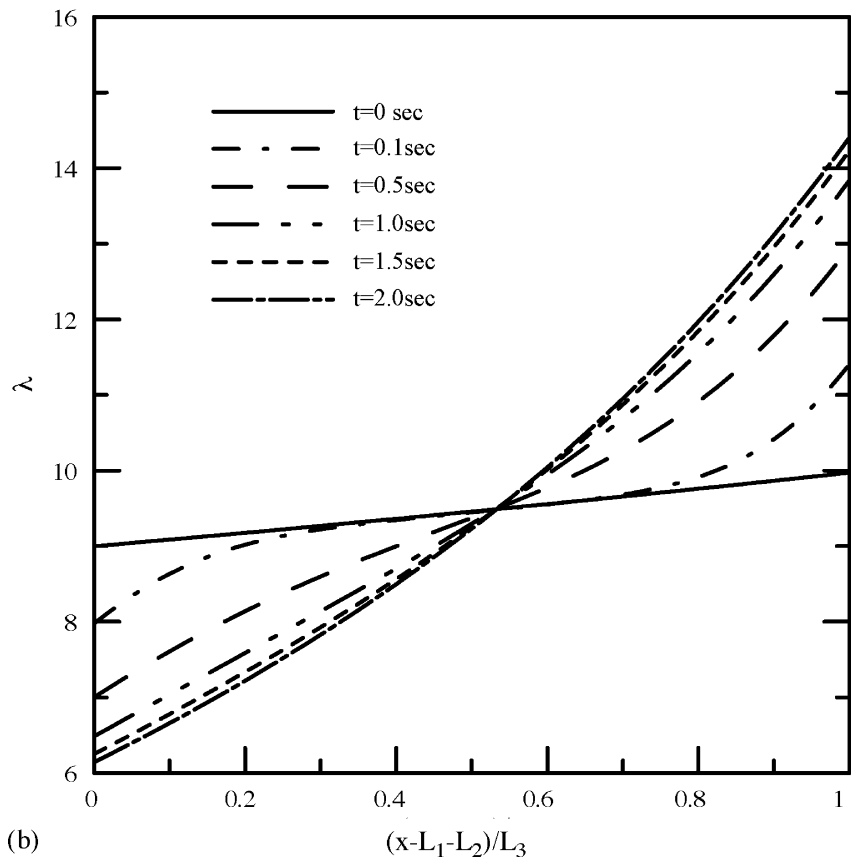

Fig. 3. Effects of humidification constant on the evolutions of the water content in PEM: (a) $k=0.01 \mathrm{~cm} \mathrm{~s}^{-1}$ and (b) $k=0.001 \mathrm{~cm} \mathrm{~s}^{-1}$.

the cathode side. As illustrated in Fig. 5(b), when the relative humidity of water vapor on the cathode side decreases to $60 \%$, the water diffuses to the cathode side in the initial stage, because the water content near the anode side is higher relative to that near the cathode side. However, as the operating current density increases with time, the water generated in reaction begins to compensate the shortage of water due to the low humidity on the cathode side in the initial stage. Then the back diffusion effects become to perform for the large water content difference between the cathode and anode sides. Consequently, the water content near the anode side rises, which in turn causes the slow 


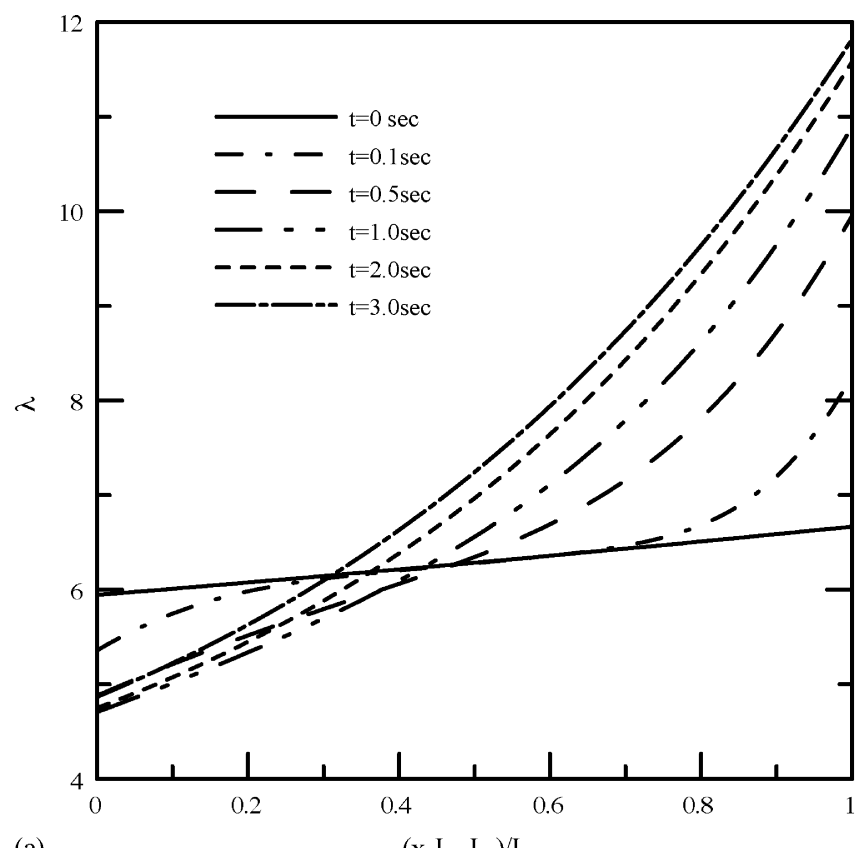

(a)

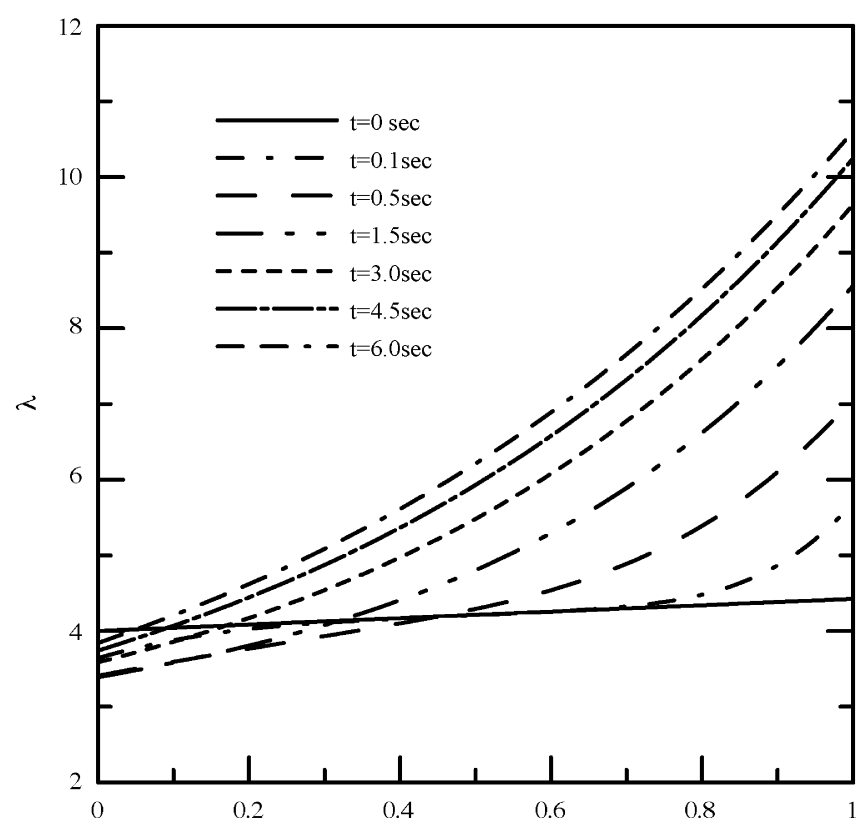

(b)

$$
\left(\mathrm{x}-\mathrm{L}_{1}-\mathrm{L}_{2}\right) / \mathrm{L}_{3}
$$

Fig. 4. Effects of humidification on both cathode and anode sides on the evolution of water content in the PEM: (a) $80 \%$ and (b) $60 \%$.

response. Therefore, the time needed for the water content in PEM to approach the steady becomes long.

Fig. 6 presents the effects of the thickness of PEM on the evolution of water content. Due to the electro-osmotic effects, with an increasing PEM thickness, the water content near the anode side of the PEM continuously rises, while the water content near the cathode side sustaining drops. As their content difference grows large enough, the back diffusion effects take place. If the thickness is too thick, the water content is not enough for the back diffusion, then the water content near the cathode side of the PEM declines. If the thickness is small, the water content in

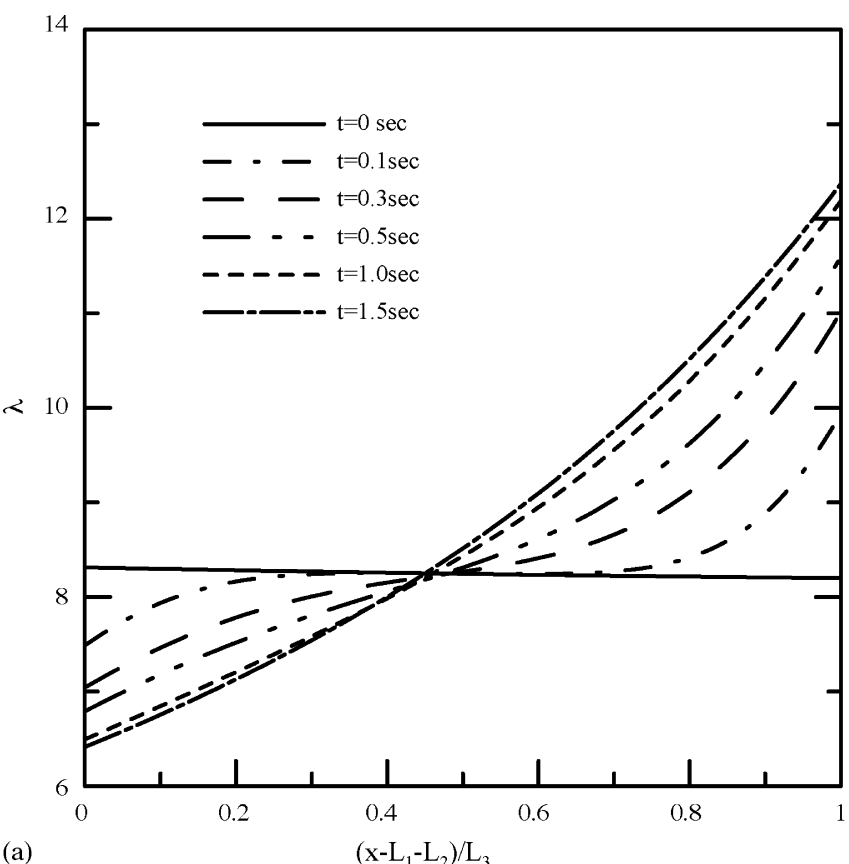

(a)

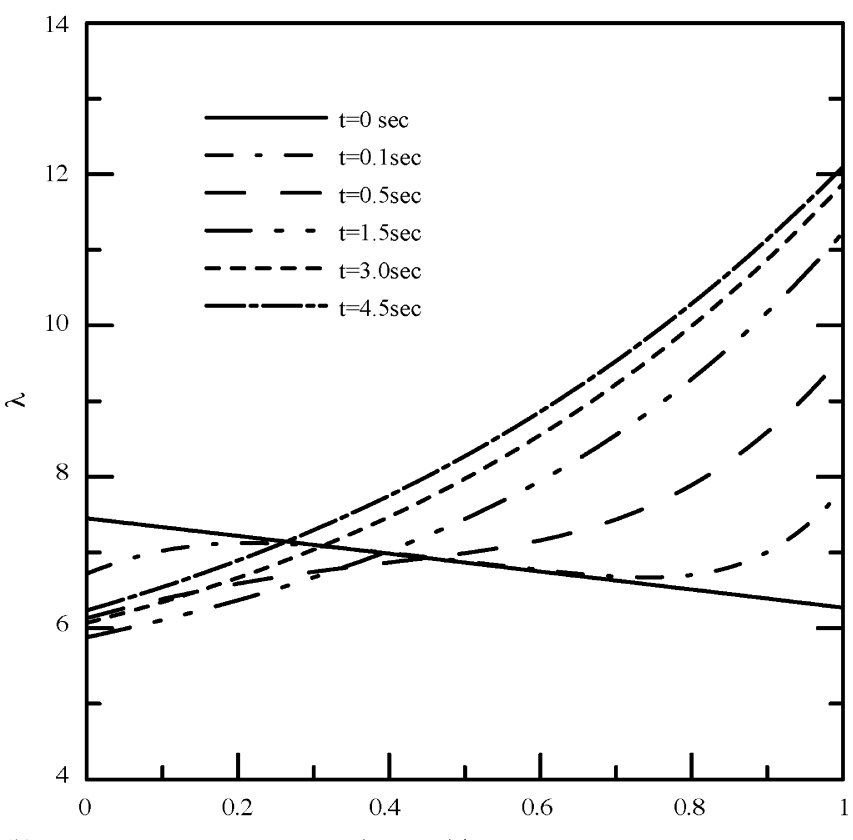

(b)

$\left(\mathrm{x}-\mathrm{L}_{1}-\mathrm{L}_{2}\right) / \mathrm{L}_{3}$

Fig. 5. Effects of humidification on the cathode side on the transient evolutions of the water content: (a) $80 \%$ and (b) $60 \%$.

PEM varies very quickly and stably, and the water distribution becomes more uniform.

Fig. 7(a) and (b) presents the response time needed for the system from one current density to another at the humidification constant $k$ of 0.01 and $0.001 \mathrm{~cm} \mathrm{~s}^{-1}$, respectively, with the relative humidity of water vapor being $100 \%$ on both cathode and anode sides. Additionally, the transverse axis denotes the initial current density, the vertical axis denotes the response time for reaching the steady state from one current density to another, and the three curves describes three different switching current densities. The comparison of Fig. 3(a) and (b) shows that, when 


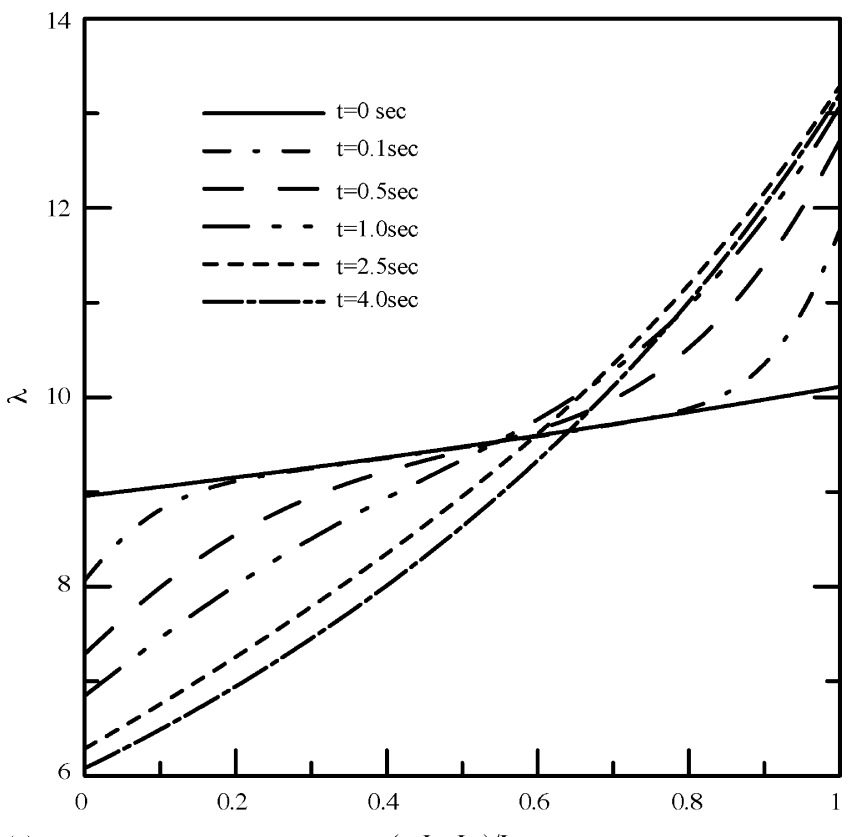

(a)

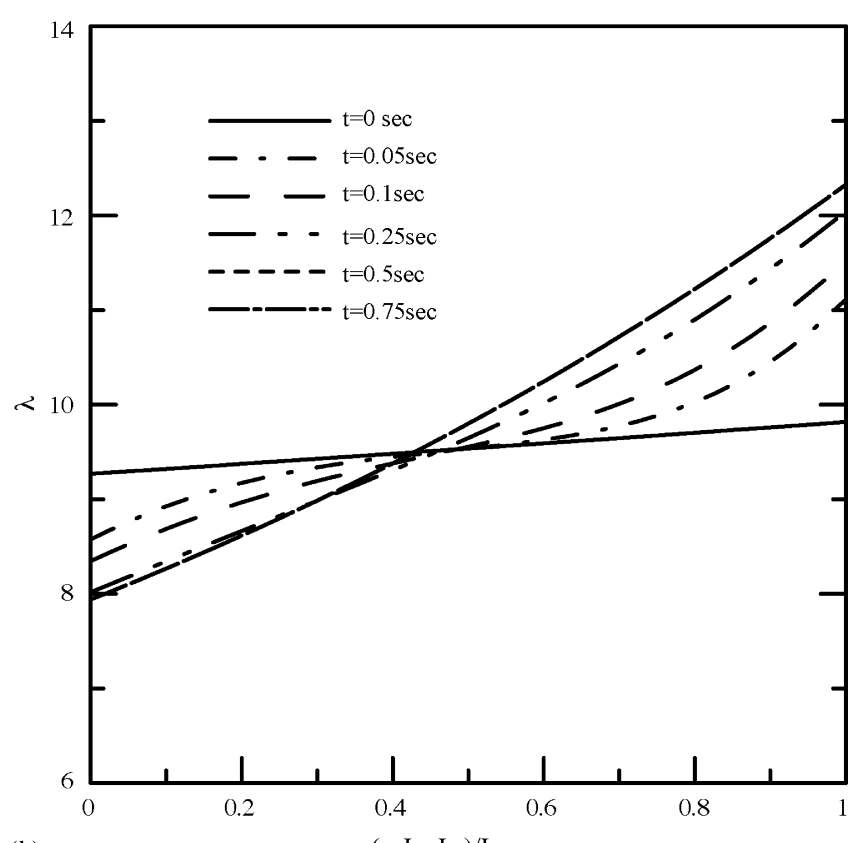

(b)

$$
\left(\mathrm{x}-\mathrm{L}_{1}-\mathrm{L}_{2}\right) / \mathrm{L}_{3}
$$

Fig. 6. Effects of thickness of PEM on the evolution of water content in PEM: (a) $L_{\mathrm{m}}=0.015 \mathrm{~cm}$ and (b) $L_{\mathrm{m}}=0.005 \mathrm{~cm}$.

$k$ is large, the response time for switching current density to $1.0 \mathrm{~A} \mathrm{~cm}^{-2}$ is longer. This is because that the water content in anode PEM is low for high current density. When $k$ is large, however, the water transport between PEM and its outside is enhanced, so that the distribution changes with little decrement, and that the time to reach the steady state is long.

Fig. 8(a) and (b) presents the response time for reaching the steady state from one current density to another with the relative humidity of water vapor on the cathode and anode sides being $80 \%$ and $60 \%$, respectively, when the humidification constant $k$ is $0.01 \mathrm{~cm} \mathrm{~s}^{-1}$, and the thickness of PEM $100 \mu \mathrm{m}$. It is found in
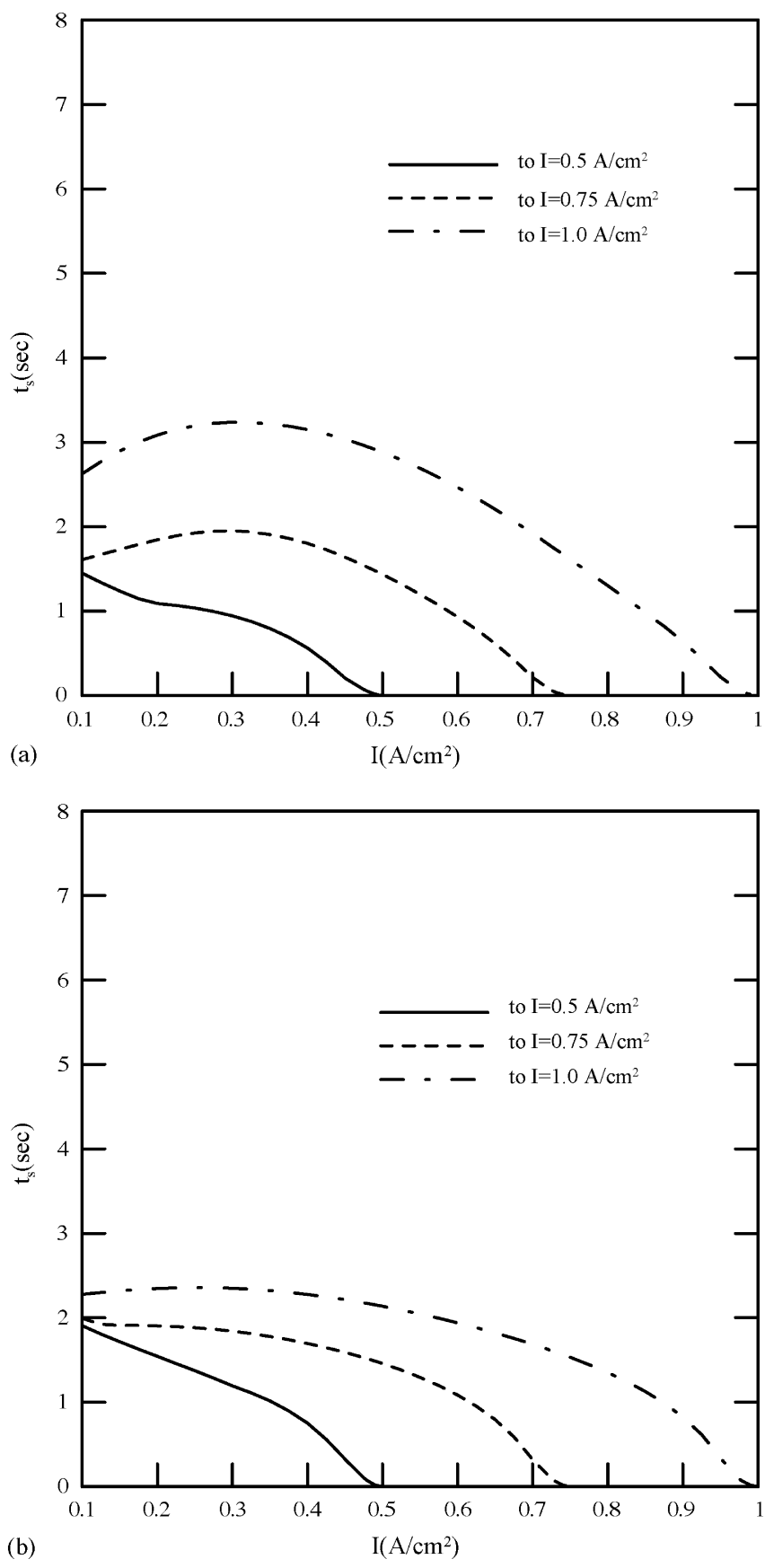

Fig. 7. Effects of humidification constant on the response time required for the PEM fuel cell reaching the steady state from initial current density $i_{0}=0.1 \mathrm{~A} \mathrm{~cm}^{-2}$ to another one: (a) $k=0.01 \mathrm{~cm} \mathrm{~s}^{-1}$ and (b) $k=0.001 \mathrm{~cm} \mathrm{~s}^{-1}$.

Fig. 8(a) that, when the humidity decreases, the response time for the PEM to reach the steady becomes long. Especially, at a low operating current density, the electro-osmotic effects are weak, and consequently the water content in PEM is mainly affected by the back diffusion process. Then the water content in anode PEM continuously rises. However, at a high operating current density, the electro-osmotic effects are enhanced, and the back diffusion effects are depressed, so that the response time for reaching the steady becomes short. Additionally, it is found in Fig. 8(b) that the response time needed to reach the steady is longer for a case with a lower humidity. The main reason is 

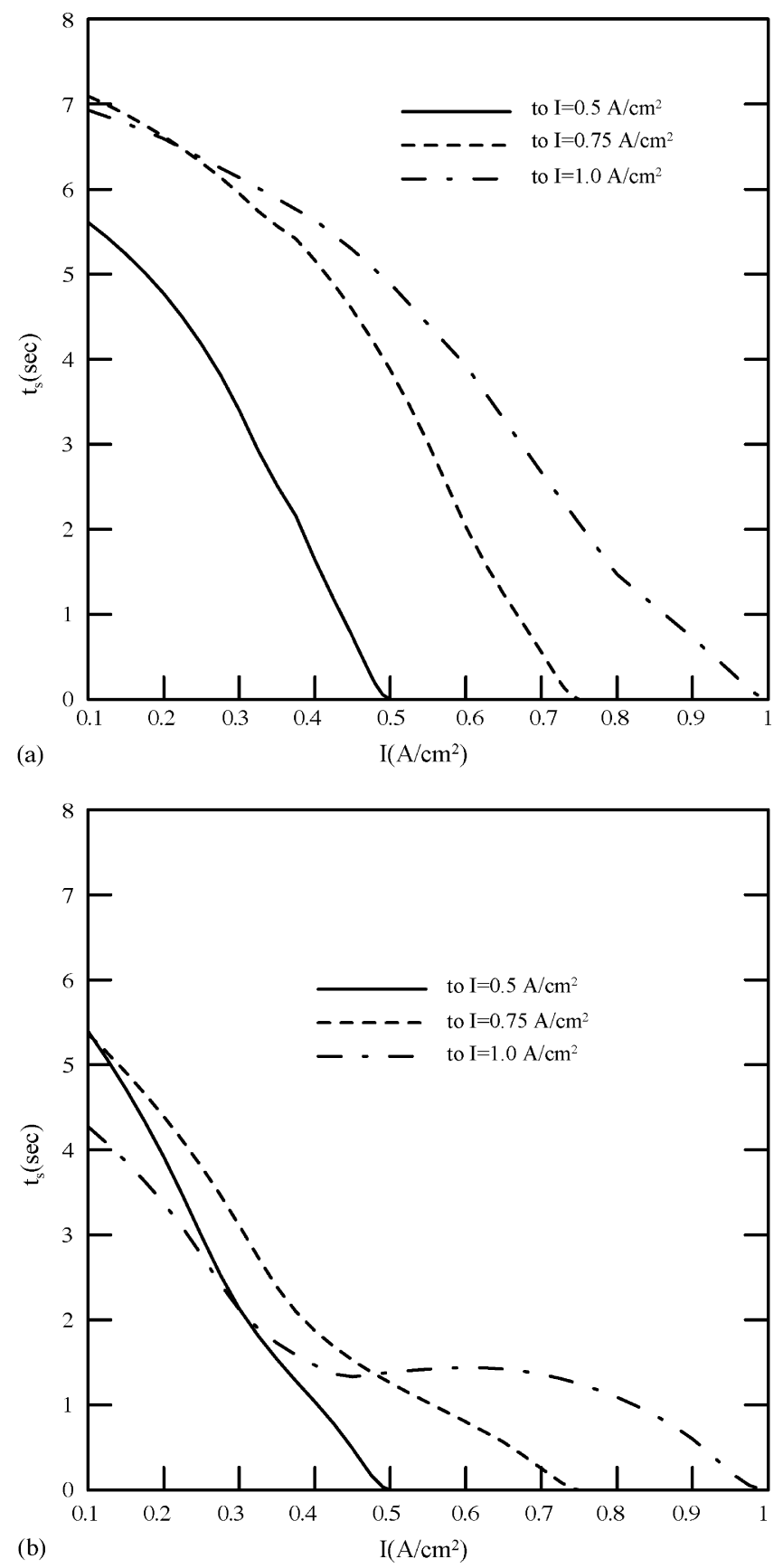

Fig. 8. Effects of humidification of both anode and cathode sides on the response time required to reach the steady state from initial current density $i_{0}=0.1 \mathrm{~A} \mathrm{~cm}^{-2}$ to another one: (a) $80 \%$ and (b) $60 \%$.

that, when the humidity on the anode side is very low, the water content difference between the two sides of PEM is so large that the water content in PEM is mainly affected by the back diffusion process, and that the response time to reach the steady is enlarged. As a conclusion, at a low humidity, the water content in PEM is insufficient, and the response time for the fuel cells to reach the water balance increases.

Fig. 9(a) and (b) presents the response time to reach the steady state from one current density to another with the relative humidity of water vapor on the cathode side being $80 \%$ and $60 \%$, respectively, when $k$ is $0.01 \mathrm{~cm} \mathrm{~s}^{-1}$, the thickness of PEM
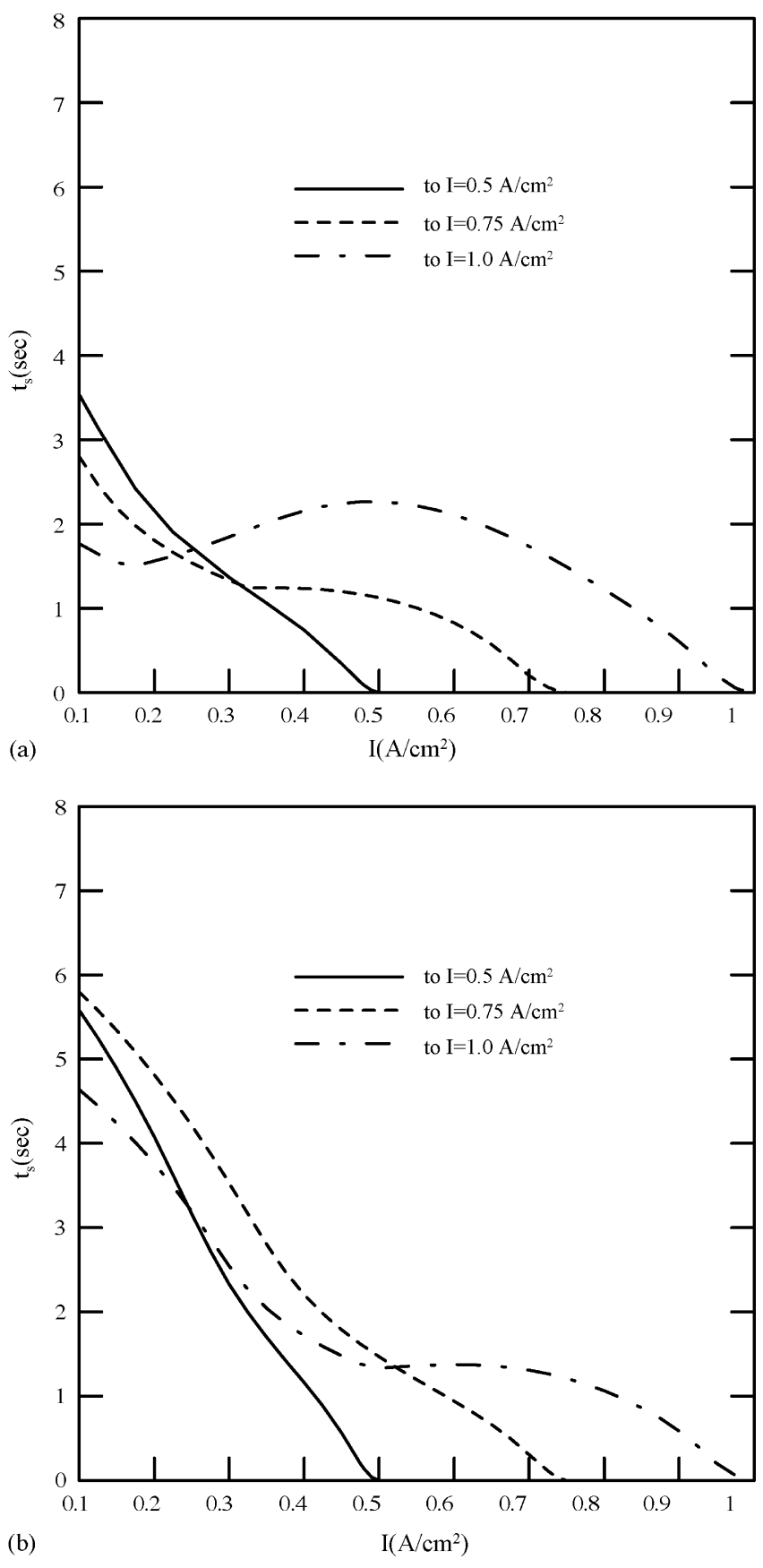

Fig. 9. Effects of humidification of cathode and anode on response time required to reach the steady state from initial current density $i_{0}=0.1 \mathrm{~A} \mathrm{~cm}^{-2}$ to another one: (a) $80 \%$ and (b) $60 \%$.

$100 \mu \mathrm{m}$. Comparing with Fig. 7(a), the response time to reach the balance is longer with switching current density to $0.5 \mathrm{~A} \mathrm{~cm}^{-2}$, while the response time with higher switching current density is shorter for a lower initial current density. It is mainly due to the weaker electro-osmotic effects at lower current density. When the back diffusion effect dominates the water content in PEM, the response time to reach the balance is enlarged. It is also found in Fig. 9(b) that the response time for the water content in PEM to reach the steady state is long when the humidity of inlet water vapor is insufficient. If the humidity of inlet water vapor on the cathode side is lower than that on the anode side, the 
response time to reach the steady state at higher current density is shorter. In addition, when the thickness of PEM is thickened, the response time for reaching the steady state increases significantly.

\section{Conclusions}

The present work investigates a one-dimensional transient process of water transport in PEM fuel cells. The diffusion and electro-osmotic effects on the water content in the PEM are explored, with only the diffusion effects are considered for the vapor liquid transport in the GDL and CL. Corresponding to the results of analysis, several conclusions are drawn as follows:

(1) The humidification constant $k$ plays an important role in the water transport. When $k$ is large, the performance of the water transport from PEM to its outside is very high, thus the water content in PEM is uniform. As $k$ decreases, the water content in cathode PEM rises due to the electroosmotic effects, and the excess water is difficult to diffuse into the cathode CL and GDL. Similarly, the supplementary rate of the water content in anode PEM is dependent upon $k$, thus the water content in anode PEM is lower for smaller $k$. The magnitude of $k$ also affects the transient behavior of water transport.

(2) When the humidity conditions on the two sides are the same, the decrease of humidity has negative effect on the water content distribution in PEM and the response time to reach the steady.

(3) When the relative humidity on the cathode decreases to $80 \%$, the steady distributions of water content in PEM under various current densities have little difference with each other. As the current density increases from 0.1 to $1.0 \mathrm{~A} \mathrm{~cm}^{-2}$, the response time to reach the steady is even shorter than that with $100 \%$ relative humidity. However, when the relative humidity in the cathode decreases to $60 \%$, the steady water content in PEM remarkably drops, and the response time to reach the steady state becomes significantly longer. Therefore, high humidity benefits the performance of fuel cells.

(4) When the PEM is thinned, the distribution of water content is more uniform, and the response time for the water content to reach the steady is apparently shorter.

\section{Acknowledgement}

The study was supported by the National Science Council, the Republic of China, through the grant NSC 94-2212-E-211-004.

\section{References}

[1] T. Okada, G. Xie, Y. Tanabe, Theory of water management at the anode side of polymer electrolyte fuel cell membranes, J. Electroanal. Chem. 413 (1996) 49-65.
[2] T. Okada, S. Møller-Holst, O. Gorseth, S. Kjelstrup, Transport and equilibrium properties of Nafion membranes with $\mathrm{H}^{+}$and $\mathrm{Na}^{+}$ions, J. Electroanal. Chem. 442 (1998) 137-145.

[3] T. Okada, G. Xie, O. Gorseth, S. Kjelstrup, N. Nakamura, T. Arimura, Ion and water transport characteristics of Nafion membranes as electrolytes, Electrochim. Acta 43 (1998) 3741-3747.

[4] T. Okada, G. Xie, M. Meeg, Simulation for water management in membranes for polymer electrolyte fuel cells, Electrochim. Acta 43 (1998) 2141-2155.

[5] T. Okada, Theory for water management in membranes for polymer electrolyte fuel cells. Part 1 . The effect of impurity ions at the anode side on the membrane performances, J. Electroanal. Chem. 465 (1999) 1-17.

[6] T. Okada, Theory for water management in membranes for polymer electrolyte fuel cells. Part 2. The effect of impurity ions at the cathode side on the membrane performances, J. Electroanal. Chem. 465 (1999) 18-29.

[7] M. Eikerling, Y.I. Kharkats, A.A. Kornyshev, Y.M. Volfkovich, Phenomenological theory of electro-osmotic effect and water management in polymer electrolyte proton-conducting membranes, J. Electrochem. Soc. 145 (1998) 2684-2699.

[8] D.M. Bernardi, M.W. Verbrugge, Mathematical model of a gas diffusion electrode bonded to a polymer electrolyte, AIChE J. 37 (1991) 1151-1163.

[9] D.M. Bernardi, M.W. Verbrugge, A mathematical model of solid-polymer-electrolyte fuel cell, J. Electrochem. Soc. 139 (1992) 2477-2490.

[10] D. Singh, D.M. Lu, N. Djilali, A two-dimensional analysis of mass transport in proton exchange membrane fuel cells, Int. J. Eng. Sci. 37 (1999) 431-452.

[11] T.E. Springer, T.A. Zawodzinski, S. Gottesfeld, Polymer electrolyte fuel cell model, J. Electrochem. Soc. 138 (1991) 2334-2342.

[12] T.E. Springer, M.S. Wilson, S. Gottesfeld, Modeling and experimental diagnostics in polymer electrolyte fuel cells, J. Electrochem. Soc. 140 (1993) 3513-3526.

[13] T.F. Fuller, J. Newman, Water and thermal management in solid-polymer-electrolyte fuel cells, J. Electrochem. Soc. 140 (1993) 1218-1225.

[14] R. Bradean, K. Promislow, B. Wetton, Transport phenomena in the porous cathode of a proton exchange membrane fuel cell, Numer. Heat Transf. A 42 (2002) 121-138

[15] T.V. Nguyen, R.E. White, A water and heat management model for proton-exchange-membrane fuel cells, J. Electrochem. Soc. 140 (1993) 2178-2186.

[16] J.S. Yi, T.V. Nguyen, An along-the-channel model for proton exchange membrane fuel cells, J. Electrochem. Soc. 145 (1998) 1149-1159.

[17] W. He, J.S. Yi, T.V. Nguyen, Two-phase flow model of the cathode of PEM fuel cells using interdigitated flow fields, AIChE J. 46 (2000) 2053-2064.

[18] W.M. Yan, C.Y. Soong, F. Chen, H.S. Chu, Effects of flow distributor geometry and diffusion layer porosity on reactant gas transport and performance of proton exchange membrane fuel cells, J. Power Sources 125 (2004) 27-29.

[19] T.A. Zawodzinski Jr., C. Derouin, S. Radzinski, R.J. Sherman, V.T. Smith, T.E. Springer, S. Gottesfeld, Water uptake by and transport through Nafion ${ }^{\circledR}$ 117 membranes, J. Electrochem. Soc. 140 (1993) 1041-1047.

[20] J.T. Hinatsu, M. Mizuhata, H. Takenaka, Water uptake of perfluorosulfonic acid membranes from liquid water and water vapor, J. Electrochem. Soc. 141 (1994) 1493-1497.

[21] P. Futerko, I.-M. Hsing, Thermodynamics of water vapor uptake in perfluorosulfonic acid membranes, J. Electrochem. Soc. 146 (1999) 2049-2053.

[22] F. Chen, Y.G. Su, C.Y. Soong, W.M. Yan, H.S. Chu, Transient behavior of water transport in the membrane of PEM fuel cell, J. Electroanal. Chem. 566 (1) (2004) 85-93.

[23] W.M. Yan, F. Chen, H.Y. Wu, C.Y. Soong, H.S. Chu, Analysis of thermal and water management with temperature-dependent diffusion effects in membrane of PEM fuel cells, J. Power Sources 129 (2) (2004) $127-137$. 